\title{
Validation of the Nuclear Cataract Grading System BCN 10
}

\author{
Rafael I. Barraquer ${ }^{a, b}$ Laura Pinilla Cortés ${ }^{a} \quad$ Miriam J. Allende ${ }^{a}$ \\ Gustavo A. Montenegro ${ }^{a}$ Bozidar Ivankovic ${ }^{c}$ Justin Christopher D'Antin ${ }^{a}$ \\ Hernán Martínez Osorio ${ }^{a}$ Ralph Michaela \\ anstitut Universitari Barraquer, Universitat Autònoma de Barcelona, and ${ }^{\mathrm{b} C e n t r o ~ d e ~ O f t a l m o l o g i ́ a ~ B a r r a q u e r, ~}$ \\ Universitat Internacional de Catalunya, Barcelona, and ' ${ }^{C}$ ospital Universitario Ramón y Cajal, Universidad de Alcalá, \\ Madrid, Spain
}

\section{Keywords}

Cataract · Grading · Validation

\section{Abstract}

Purpose: To evaluate a new nuclear cataract grading system which is intended as a surgical guidance system to predict lens hardness before cataract surgery. Methods: The new BCN 10 grading system consists of frontal and cross-sectional slit-lamp images of human eye lenses, ranging from a completely transparent lens nucleus to a totally black nuclear cataract. Validation was done with 9 observers for 110 cases. Two modalities were applied, and observers were asked to use only whole digits and then half digits for grading. $\boldsymbol{R} \boldsymbol{e}$ sults: Repeatability with regard to test-retest differences showed a mean limit of agreement of 1.70 for whole digits and 1.32 for half digits. The absolute test-retest difference was close to zero for low as well high degrees of cataracts. Reliability for the entire group of 9 observers yielded an intraclass correlation coefficient which was within the same confidence interval, i.e., 0.991-0.995, for whole digits and half digits. Conclusions: BCN 10 grading repeatability was not affected by the severity of the cataract. It showed very good repeatability. Repeatability was significantly higher when the observers used half digits compared to whole digits. Reliability was found to be very good as well, independently of the use of whole or half digits.

(C) 2017 The Author(s)

Published by S. Karger AG, Basel

\section{KARGER}

E-Mail karger@karger.com www.karger.com/ore

This article is licensed under the Creative Commons AttributionNonCommercial-NoDerivatives 4.0 International License (CC BYNC-ND) (http://www.karger.com/Services/OpenAccessLicense). Usage and distribution for commercial purposes as well as any distribution of modified material requires written permission.

\section{Introduction}

The development of photographic standards for the grading of cataracts has facilitated the assessment of opacities and coloration in the crystalline lens. However, in clinical application, there will always be some degree of subjectivity because it will depend on the judgment of the examiner.

These grading systems were designed to be easy to learn and apply and to be reproducible. Different classification systems of cataracts have been proposed, with different levels of complexity depending on the applications for which they were designed (i.e., diagnosis, epidemiological studies, analysis of risk factors, studies of potential anticataract drugs, and presurgical assessment).

A grading scale can be defined as a tool that enables quantification of the severity of a condition with reference to a set of standardized descriptions or illustrations [1]. Currently, rather than applying descriptive or qualitative terms, such as incipient, mild, severe, or mature, to indicate a stage of development of cataracts, numeric scaling or grading systems are often used. The clinician makes an observation and assigns a numeric value, and this number serves as a reference based on which any future change may be judged.

Ralph Michael

Institut Universitari Barraquer

Laforja 88

ES-08021 Barcelona (Spain)

E-Mail ralphm@barraquer.com 
The handicap of using a grading scale is that we have to divide the continuous process of cataract development into discrete groups. This could lead to a scale that may be too coarse. When a scale is too coarse the concordance tendency is higher but the sensitivity is lower. Using a finer scale can substantially enhance the ability of the clinician or researcher to detect small changes $[2,3]$. On the other hand, some studies have observed that, even when the scale allows for increments of 0.1 , there is a tendency for observers to mainly classify the changes using increments of 1 or $0.5[1,3]$.

The Oxford system [4] is an example of a complex grading system in which a large number of cataract characteristics need to be analyzed; this includes projection of a resolution target with an ophthalmoscope and slit-lamp evaluation for cortical and nuclear layers, which includes different cataract morphologies like vacuoles, retro dots, focal dots, nuclear brunescence, and white nuclear scatter. It is a very detailed and complex classification but it is difficult to apply clinically. Other proposed systems, simpler in terms of design, implementation, and reproducibility, have been designed primarily for epidemiological studies. One example is the Japanese Cooperative Cataract Epidemiology Study Group System [5]. It is also based on standardized images and was designed to be simple and easy to use, sacrificing accuracy and the ability to detect small variations. Another example is the WHO Simplified Cataract Grading System [6], an initiative of the World Health Organization. Their purpose was to unify and simplify the criteria used in several other classifications. The most widely used system today is the Lens Opacities Classification System III (LOCS III) [7], which is a chart consisting of 6 slit-lamp images for grading nuclear color and nuclear opalescence, 5 retroillumination images for grading cortical cataracts, and 5 retroillumination images for grading posterior subcapsular cataracts.

Our proposed grading system BCN 10 is intended as a clinical and surgical guidance system. It focuses primarily on the nucleus, because it is the most critical component for predicting surgical needs and choosing the most appropriate surgical technique according to the hardness of the nucleus. A special emphasis has been made on more advanced stages of cataracts, which are precisely the ones that are more difficult to operate on. In this respect, the LOCS III has certain limitations due to the fact that it does not include the more advanced grades of cataracts.

In the present study, we validate our nuclear cataract grading system $\mathrm{BCN} 10$.

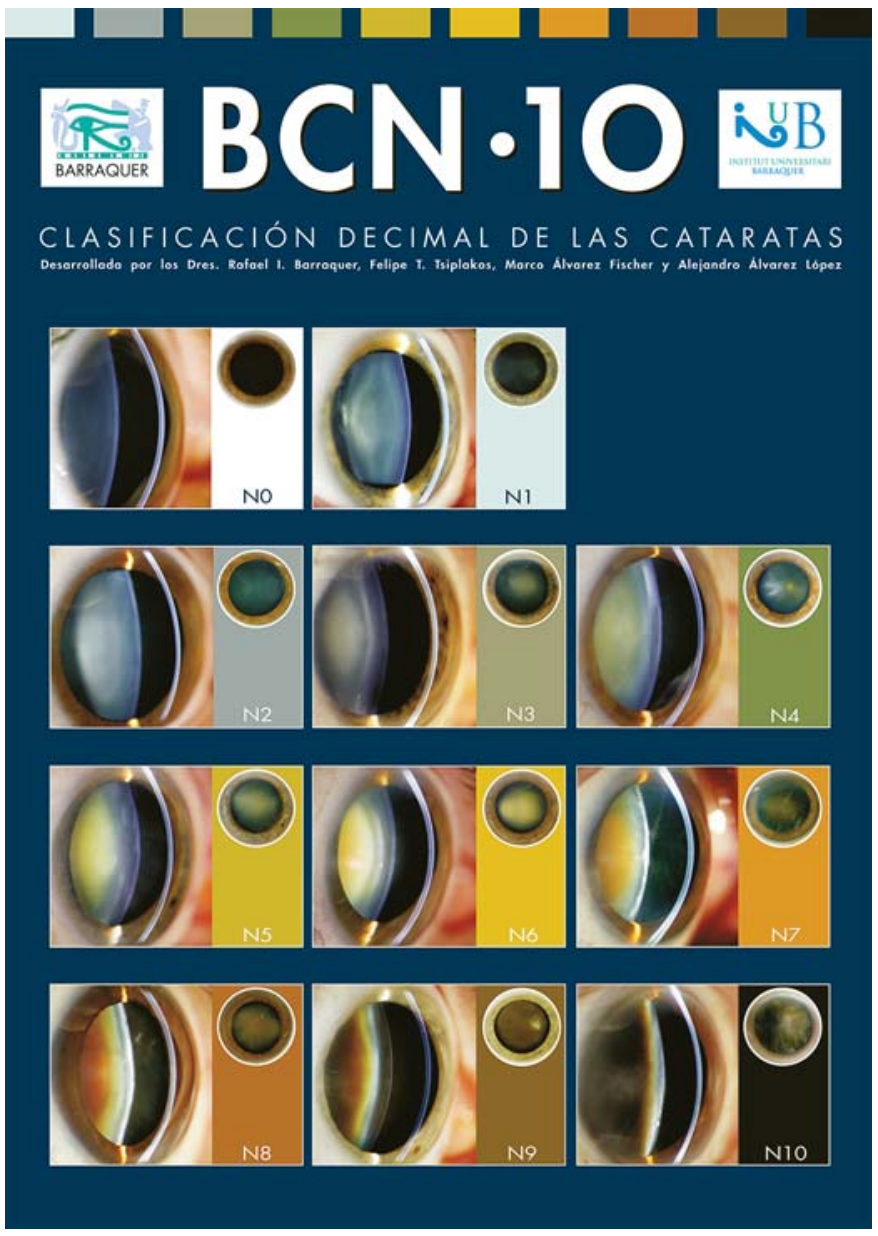

Fig. 1. BCN 10 nuclear grading system chart showing a slit-lamp cross-sectional image, a smaller frontal view image, and the relative color for each stage of cataract development (N0 to N10).

\section{Methods}

The BCN 10 Nuclear Grading System

The BCN 10 grading system is provided as an A4-sized laminated chart with high-resolution photographs. These photographs were taken by photography staff using a Zeiss slit lamp (Carl Zeiss AG; Oberkochen, Germany) with the following settings: beam width, $10 \mathrm{~mm}$; height for a frontal view, $10 \mathrm{~mm}$; and height and width at $45^{\circ}$ for a cross-sectional view, 10 and $1 \mathrm{~mm}$, respectively.

The system divides the nuclear cataract progression into a baseline clear lens (N0) and 10 grades of opacification (N1 to N10). This degree of opacity is our grading scale unit. The grading system chart shows a large slit-lamp cross-sectional image, a smaller frontal view image, and the relative color for each stage of cataract development (Fig. 1). The grades were chosen in equidistant intervals from a normal aged crystalline lens (N1) to a completely dark lens (cataracta nigra - N10).
Barraquer et al. 
Table 1. ICC for each observer (repeatability) and for the entire group (reliability) using whole and half digits for the nuclear cataract grading system $\mathrm{BCN} 10$

\begin{tabular}{lll}
\hline & Whole digits & Half digits \\
\hline $\begin{array}{l}\text { Repeatability } \\
\text { Observer }\end{array}$ & \\
A & & \\
$\mathrm{B}$ & 0.934 & 0.963 \\
$\mathrm{C}$ & 0.957 & 0.963 \\
$\mathrm{D}$ & 0.974 & 0.977 \\
$\mathrm{E}$ & 0.955 & 0.964 \\
$\mathrm{~F}$ & 0.940 & 0.979 \\
$\mathrm{G}$ & 0.880 & 0.955 \\
$\mathrm{H}$ & 0.959 & 0.976 \\
$\mathrm{I}$ & 0.980 & 0.979 \\
Mean (A to I) & 0.981 & 0.982 \\
\hline Reliability & $0.951^{\mathrm{a}}$ & $0.971^{\mathrm{a}}$ \\
Entire group & & \\
Confidence interval & $0.993^{\mathrm{b}}-0.995$ & $0.994^{\mathrm{b}}$ \\
\hline
\end{tabular}

All single ICC were significant according to F-tests $(p<0.001)$. Repeatability ICC were normally distributed according to a Shapiro-Wilk test. ICC, intraclass correlation coefficient. ${ }^{\text {a }}$ The mean repeatability ICC using whole and half digits were significantly different according to a paried $t$ test $(p=0.043)$. ${ }^{\mathrm{b}}$ The reliability ICC were not significantly different using whole and half digits as shown by the identical confidence intervals.

\section{Validation Process}

The basis for the validation process was standardized photographs from 110 patients scheduled for cataract surgery at the Centro de Oftalmología Barraquer. Patients were selected in order to cover all degrees of cataract equally. This included one frontal view of the eye and a cross-sectional view, both with dilated pupils, taken at the slit lamp by the photography staff of the clinic. The photographs were presented at random on a computer display and had to be compared with the BCN 10 chart on the side.

Observers for the validation process were 3 ophthalmic surgeons and 6 residents in ophthalmology (at the end of their training). The validation process was done for 2 different modalities, first allowing the use of only 1-grade increments (whole digits), yielding 11 grading steps, and about 6 months later allowing 0.5-grade increments (half digits), yielding a total of 21 grading steps.

The grading for both modalities was done twice with an interval of about 3 weeks to evaluate the repeatability of the grading results. Repeatability, or intraobserver agreement, means the ability of the grader to give similar results for a repeated test (test-retest) under the same conditions. We also evaluated the reliability or precision of the grading system, which is the accuracy of all of the observers in giving the same value to the same object of evaluation. Reliability can be referred to as interobserver agreement $[8,9]$.

Statistical Analysis

We consider our grading scale unit (degree of opacity) to be continuous and quantitative data. The grades of the BCN 10 chart have been chosen in equidistant intervals and are evaluated with 11 or 21 grading steps.

The Bland-Altman approach [10] was used to evaluate repeatability. It applies a diagram which shows the difference or discrepancy between 2 repeated observations plotted against the mean of both observations. The standard deviation of all the differences multiplied by 1.96 gives the so-called limits of agreement or coefficient of repeatability $[2,3,10]$. The values of the limits of agreement are in grading scale units (degrees of opacity). The lower the value of the limit of agreement, the better the repeatability.

The intraclass correlation coefficient (ICC) [11] was applied to evaluate repeatability as well as reliability. The ICC estimation is based on analysis of variance techniques. It can very between 0 and 1. The maximum value is 1 when all observers assign the same grade. It should be greater than 0.7 in order for the grading system to be considered reliable [12].

We calculated the ICC using SPSS version 13.0 (SPSS Inc., Chicago, IL, USA) choosing the Statistical Reliability analysis option with the model Alfa. Based on the fact that we had a sample group of observers and cases, we used the Two-Way Random model and selected absolute agreement. The Single Measures results were used to evaluate repeatability, and the Average Measures were used for reliability.

The significance level and confidence coefficients were set to 0.05 and 0.95 , respectively.

\section{Results}

The test-retest responses of the 9 observers for the 110 cases for both modalities, i.e., whole digits and half digits, were evaluated for repeatability. The mean discrepancy between the two repeated tests was close to zero for all observers, ranging between -0.308 and +0.514 for whole digits and between -0.311 and +0.261 for half digits. The limits of agreement ranged from 1.09 to 2.78 for whole digits and from 0.94 to 1.70 for half digits (Fig. 2a, b). A paired $t$ test revealed that the mean limit of agreement using whole digits (i.e., 1.70) was significantly higher compared to the value using half digits (i.e., 1.32), with $p=$ 0.008 .

We also pooled all 990 repeated observations from our 9 observers together in one Bland-Altmann plot (Fig. 2c, d). Figure 2 shows that all grades of opacification from N0 to N10 were present in a balanced manner. The discrepancy between test and retest values was close to zero for low as well for high degrees of cataracts. The combined mean discrepancy was -0.033 and -0.062 and the combined limit of agreement was 1.81 and 1.38 for whole and half digits, respectively (Fig. 2c, d).

The ICC for observer repeatability was lower for whole digits (mean 0.951, range $0.880-0.981$ ) as compared to half digits (mean 0.971, range 0.955-0.982). This difference was statistically significant $(p=0.043$; Table 1$)$. 


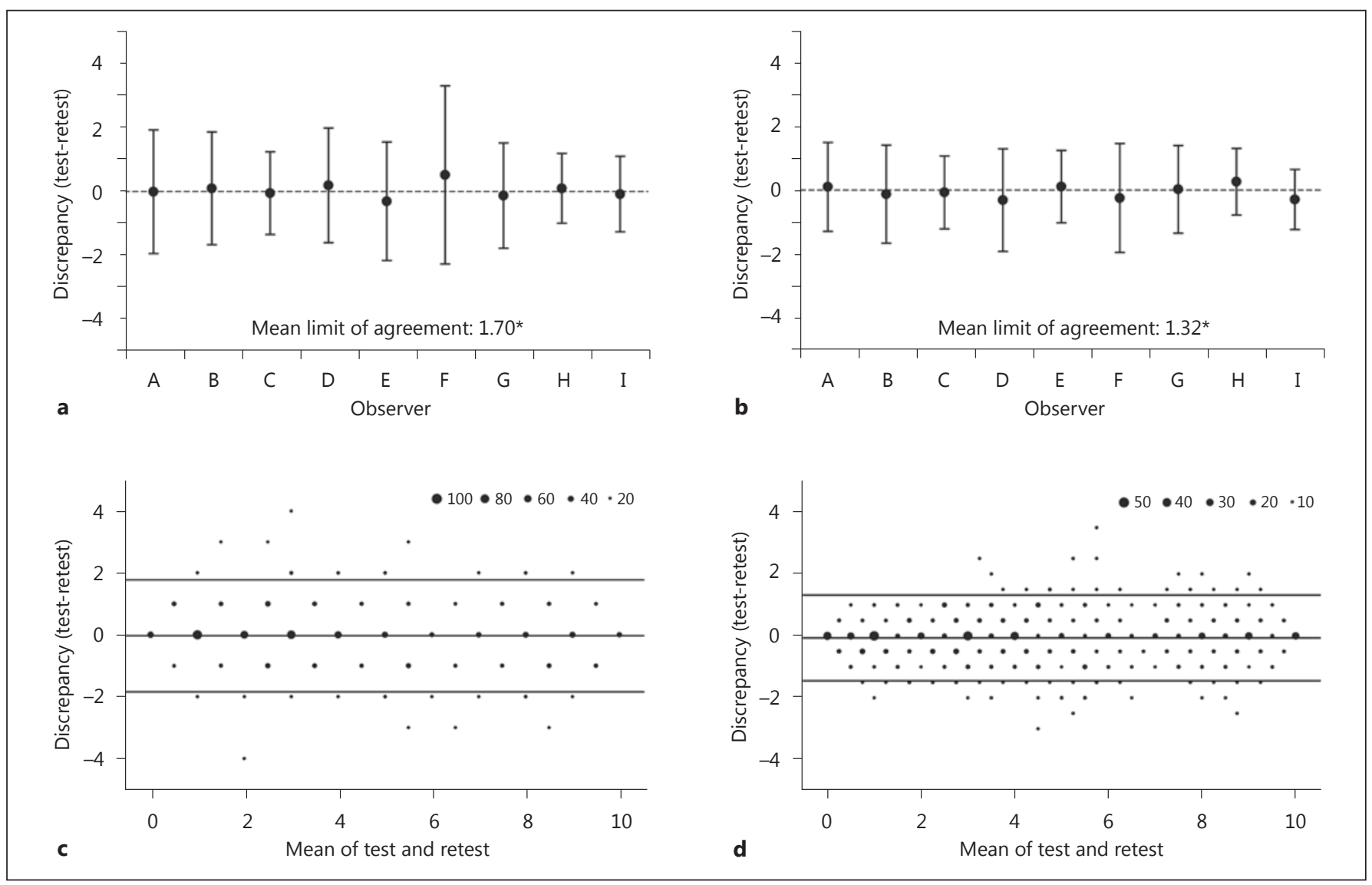

Fig. 2. a, b Mean test-retest difference (discrepancy) with corresponding limits of agreement for all individual observers grading 110 cases, applying 2 modalities: use of only whole digits (a) or half digits (b) for grading. The dashed line indicates zero discrepancy. The asterisk indicates a significant difference of the mean limits of

The ICC for reliability, considering all 9 observers together, was very high (0.993 and 0.994$)$ but not different for whole and half digits as shown by the confidence interval of the ICC, which was between 0.991 and 0.995 for both grading modalities (Table 1).

\section{Discussion}

The BCN 10 grading system was designed to be used at the slit lamp with the patient in front. However, for the validation process it was not practical for all 9 observers to have the same 110 patients personally in front of a slit lamp. Therefore, validation was done with the help of standardized photographs on the same type of computer screen. This might have introduced some additional variation with respect to having the patient in front of the slit lamp. agreement comparing the use of whole and half digits. $\mathbf{c}, \mathbf{d}$ BlandAltman plots for all test-retest discrepancies from all 9 observers pooled together for whole (c) and half digits (d). Horizontal lines indicate the overall mean discrepancy and the respective limits of agreement calculated for all observers together.

There is a continuous debate about which metric and statistical test to use for quantification and comparison of repeatability and reliability. The Bland-Altman approach is straightforward and focuses on the difference between observations. The ICC focuses, as the name implies, on correlations. It quantifies the correlation between observers very well, but it is insensitive to systematic errors. We used both approaches in our validation analysis.

The BCN 10 grading system for nuclear cataracts yielded very good repeatability in terms of correlation, with a mean ICC of 0.951 for whole digits and 0.971 for half digits. A grading system is considered to give excellent results when the ICC is greater than 0.75 [12].

In absolute terms, our system gave a repeatability confidence, or limit of agreement, of 1.70 grading scale units for whole digits and 1.32 for half digits. This is compared to 2.0 grading scale units reported for the LOCS II (whole 
digits) and 0.7 for the LOCS III (decimal digits) for nuclear color and opalescence [7]. One has to consider that the LOCS uses a scale from 0 to 6 and in the LOCS III the observer sees images for 5 grades of opacity (a clear lens image is missing) and is asked to grade the cataract with 0.1 increments (interpolating between the images representing whole digits). Such small increments of 0.1 are known to provide narrower limits of agreement [1]. On the other hand, some studies have observed that, even when the scale allows for increments of 0.1 , there is a tendency for the rater to mainly classify the changes using increments of 1 or $0.5[1,3]$. This is why we asked observers in our second modality to use half digits. We think that this resulted in a reasonable compromise between practical application and relatively low limits of agreement of 1.32 grading scale units, considering our scale from 0 to 10 .

Automated optical devices such as the Pentacam (Oculus GmbH, Wetzlar-Dutenhofen, Germany) or the Optical Quality Analysis System (Visiometrics SL, Tarrasa, Spain) struggle to give accurate results in cases of severe cataracts (e.g., BCN 10 grades above 6). These objective devices rely on the transmission of light through the crystalline lens. Scheimpflug systems analyze the increase in backscatter as cataracts progress; however, advanced cataracts present less backscatter because light is absorbed. Double pass systems have to pass the measuring light through the lens twice. In very opaque lenses this will not work properly. Notably, to our knowledge, these automated optical devices have only been tested in cases of mild to moderate cataracts (up to LOCS III grade 4) [1315].

Our system could be especially useful in developing countries due to their lack of access to these objective test devices. These countries also have a prevalence of highergrade cataracts, which other image-based grading systems do not take in to account [16].

The grading repeatability for BCN 10 was not affected by the severity of the cataract, because we found that the absolute test-retest differences were close to zero for low as well for high degrees of cataracts (Fig. 2a, b).

The reliability of BCN 10 for the entire group of $9 \mathrm{ob}-$ servers gave an ICC which was within the same confidence interval, i.e., 0.991-0.995, for whole digits and for half digits. This represents excellent reliability and the fact that it is the same for both modalities gives the user the option to apply whole or half digits, though we would recommend half digits because of the better repeatability.

\section{Disclosure Statement}

The authors have no proprietary interests in the materials described in this article and they have no conflicts of interests to declare.

\section{References}

1 Efron N, Morgan PB, Katsara SS: Validation of grading scales for contact lens complications. Ophthalmic Physiol Opt 2001;21:1729.

2 Bullimore MA, Bailey IL: Considerations in the subjective assessment of cataract. Optom Vis Sci 1993;70:880-885.

3 Bailey IL, Bullimore MA, Raasch TW, Taylor HR: Clinical grading and the effects of scaling. Invest Ophthalmol Vis Sci 1991;32:422-432.

4 Sparrow JM, Bron AJ, Brown NA, Ayliffe W, Hill AR: The Oxford Clinical Cataract Classification and Grading System. Int Ophthalmol 1986;9:207-225.

5 Sasaki K, Shibata T, Obazawa H, Fujiwara T, Kogure F, Obara Y, Itoi M, Katou K, Akiyama K, Okuyama S: Classification system for cataracts: application by the Japanese Cooperative Cataract Epidemiology Study Group. Ophthalmic Res 1990;22(suppl 1):46-50.

6 Thylefors B, Chylack LT Jr, Konyama K, Sasaki K, Sperduto R, Taylor HR, West S: A simplified cataract grading system. Ophthalmic Epidemiol 2002;9:83-95.
7 Chylack LT Jr, Wolfe JK, Singer DM, Leske MC, Bullimore MA, Bailey IL, Friend J, McCarthy D, Wu SY: The Lens Opacities Classification System III. The Longitudinal Study of Cataract Study Group. Arch Ophthalmol 1993;111:831-836.

8 Wong AL, Leung CK, Weinreb RN, Cheng AK, Cheung CY, Lam PT, Pang CP, Lam DS: Quantitative assessment of lens opacities with anterior segment optical coherence tomography. Br J Ophthalmol 2009;93:61-65.

9 Chong E, Simpson T, Fonn D: The repeatability of discrete and continuous anterior segment grading scales. Optom Vis Sci 2000;77: 244-251.

10 Bland JM, Altman DG: Statistical methods for assessing agreement between two methods of clinical measurement. Lancet 1986;1:307310.

11 Koch GG: Intraclass correlation coefficient; in Kotz S, Johnson NL (eds): Encyclopedia of Statistical Sciences 4. New York, Wiley, 1982, pp 213-217.
12 Zaki R, Bulgiba A, Nordin N, Azina IN: A systematic review of statistical methods used to test for reliability of medical instruments measuring continuous variables. Iran J Basic Med Sci 2013;16:803-807.

13 Pan AP, Wang QM, Huang F, Huang JH, Bao FJ, Yu AY: Correlation among Lens Opacities Classification System III grading, Visual Function Index-14, Pentacam nucleus staging, and objective scatter index for cataract assessment. Am J Ophthalmol 2015;159:241247.

14 Cochener B, Patel SR, Galliot F: Correlational analysis of objective and subjective measures of cataract quantification. J Refract Surg 2016; 32:104-109.

15 Artal P, Benito A, Perez GM, Alcon E, De Casa A, Pujol J, Marin JM: An objective scatter index based on double-pass retinal images of a point source to classify cataracts. PLoS One 2011;6:e16823.

16 Lam D, Rao SK, Ratra V, Liu Y, Mitchell P, King J, Tassignon MJ, Jonas J, Pang CP, Chang DF: Cataract. Nat Rev Dis Primers 2015; $1: 15014$

Validation of the BCN 10

Ophthalmic Res 2017;57:247-251 